\title{
Observations of linear and nonlinear processes in the foreshock wave evolution
}

\author{
Y. Narita ${ }^{1}$, K.-H. Glassmeier ${ }^{1,2}$, M. Fränz ${ }^{2}$, Y. Nariyuki ${ }^{3}$, and T. Hada $^{3}$ \\ ${ }^{1}$ Institute of Geophysics and Extraterrestrial Physics, Technical University of Braunschweig, Mendelssohnstr. 3, 38106 \\ Braunschweig, Germany \\ ${ }^{2}$ Max-Planck-Institute for Solar System Research, Max-Planck-Str. 2, 37191 Katlenburg-Lindau, Germany \\ ${ }^{3}$ Department of Earth System Science and Technology, Kyushu University, 816-8580, Kasuga, Japan
}

Received: 28 March 2007 - Revised: 2 July 2007 - Accepted: 2 July 2007 - Published: 9 July 2007

\begin{abstract}
Waves in the foreshock region are studied on the basis of a hypothesis that the linear process first excites the waves and further wave-wave nonlinearities distribute scatter the energy of the primary waves into a number of daughter waves. To examine this wave evolution scenario, the dispersion relations, the wave number spectra of the magnetic field energy, and the dimensionless cross helicity are determined from the observations made by the four Cluster spacecraft. The results confirm that the linear process is the ion/ion righthand resonant instability, but the wave-wave interactions are not clearly identified. We discuss various reasons why the test for the wave-wave nonlinearities fails, and conclude that the higher order statistics would provide a direct evidence for the wave coupling phenomena.
\end{abstract}

\section{Introduction}

\subsection{Origin of foreshock waves}

The physics of the collisionless shock waves is one of the most interesting subjects in space plasma. While the shock waves in the ordinary gas dynamics dissipate the kinetic energy of the supersonic flow into the heat on a scale within a few mean free paths of particles, the collisionless shock waves exhibit a variety of dissipation mechanisms in a dilute plasma. When the flow speed is sufficiently large compared to the Alfvén speed, the collisionless shock waves specularly reflect a portion of the incoming charged particles (supercritical shock). The reflected particles either gyrate about the magnetic field just in front of the shock wave when it is quasi-perpendicular to the shock normal direction, or stream toward upstream along the magnetic field when the field is quasi-parallel to the shock normal. In the latter case the reflected particles form a field-aligned beam against the incoming flow. Although both the ions and the electrons can be reflected at the shock, the ions play more important roles in the shock dissipation. This is because the ions carry the most of energy and momentum in the plasma. The backstreaming ions "warn" the upstream plasma about the existence of the shock wave, and brake the upstream flow a little before it reaches the shock wave. The back-streaming ions encounter the incoming ion population and they form an unstable beam-beam velocity distribution (the incoming and the reflected ions). The upstream plasma is therefore subject to waves and turbulence in order to relax the unstable velocity distribution. The region where the back-streaming ions exist is called the foreshock, which is often accompanied by fluctuations of the magnetic field and the plasma. The foreshock itself belongs to one of the dissipation mechanisms of the collisionless shock waves and provides pre-thermalization processes before the plasma stream reaches the shock wave.

Some hints about the wave processes in the foreshock are already given by the dispersion relations and the wave number spectra which are experimentally determined on the basis of the four point measurements in space. (Narita et al., 2003, 2006; Narita and Glassmeier, 2005). The results of the dispersion analysis imply that a certain linear, micro-instability process is operating and reorganizing the beam-beam velocity distribution. On the other hand turbulence-like energy spectra are identified, suggesting that some nonlinear, wavewave interaction processes be also present. Yet it is not clear how these processes are related to each other, or under what conditions the transition from the linear process to the nonlinear process takes place. We therefore put a hypothesis about the evolution of the foreshock waves. It consists of two stages as follows.

Correspondence to: Y. Narita

(y.narita@tu-bs.de)

Published by Copernicus Publications on behalf of the European Geosciences Union and the American Geophysical Union. 


\subsection{Stage 1 - parent wave excitation}

We assume that the primary waves are excited by the ion/ion right-hand resonant instability for the following reasons. In the linearized Vlasov equation model, the field-aligned ion beam injected into a plasma is subject to three kinds of electromagnetic micro-instabilities: right-hand resonant, lefthand resonant, and non-resonant ion/ion instabilities. Here the term "ion/ion" means the core ions and the beam ions. These instabilities are well documented by Gary (1993). These instabilities are linear in the sense that the fluctuation amplitude (or the envelope of the fluctuation) grows exponentially as a function of time.

The right-hand instability stems from a resonance between the ion beam and the right-hand circularly polarized wave and it is the fastest growing under typical foreshock parameters. The instability excites the waves propagating along the magnetic field. The waves follow the magnetosonic/whistler branch in the dispersion relation, which becomes the Alfvén waves at the small wave number limit. Here we mean the Alfvén waves by the ones that satisfy the dispersion relation $\omega=k_{\|} V_{A}$, where $\omega$ denotes the frequency, $k_{\|}$the wave number parallel to the mean magnetic field, and $V_{A}$ the Alfvén speed. The maximum growth rate is typically at the wave number $\left|k_{\|} V_{A} / \Omega_{p}\right| \approx 0.1$, where $\Omega_{p}$ denotes the ion cyclotron frequency. We assume protons for the ions (subscript $p$ ). In observations the waves are often termed as the Alfvén waves, as they propagate along the background magnetic field. The experimentally determined dispersion relations confirm the magnetosonic/whistler branch (Narita et al., 2003; Narita and Glassmeier, 2005).

The resonance between the left-hand polarized waves and the ion beam is also possible, but it is easier to excite the right-hand mode under cool beam conditions because at the low thermal velocities of the beam there are only few ions which can resonate with the left-hand mode. While the righthand and the left-hand resonant modes excite waves parallel to the ion beam direction, the non-resonant mode excites waves in the opposite direction. The non-resonant mode is basically a firehose instability, caused by the inertia of the fast ion beam exerting a centrifugal force on the bent magnetic field. This mode has a larger threshold to grow, since it has to overcome the restoring forces of perpendicular pressure.

\subsection{Stage 2 - daughter wave excitation}

When the primary wave amplitude exceeds a certain threshold, excess power spills into the daughter waves at the expense of the primary wave. If the amplitude is increased further, the daughter waves generate further daughter waves successively. Hence doubling the wave amplitude may not be stable any more but result in a cascade of daughter waves. Such a process represents the wave-wave nonlinearities and is referred to as the parametric instabilities.
The parametric instabilities themselves exhibit different processes between the high and the low $\beta$ regimes, where $\beta=p_{t h} / p_{m}$, the ratio of the thermal to the magnetic pressure. The decay of circularly polarized, parallel-propagating Alfvén waves with respect to the mean magnetic field through the nonlinear interaction was first suggested by Galeev et al. (1963) and Sagdeev and Galeev (1969), where a parent Alfvén wave collapses into plasma density fluctuations and a backward-propagating daughter Alfvén wave (decay instability). This process was studied in detail using dispersion relations for ideal magnetohydrodynamics (MHD) (Derby, 1978; Goldstein, 1978). However, Alfvén waves are also subject to "modulational instability", where all the daughter waves propagate in the same direction as the parent wave (Mio et al., 1976; Mjølhus, 1976; Nariyuki and Hada, 2006a). These two instability processes have been systematically studied in the framework of the Hall-MHD (Longtin and Sonnerup, 1986; Terasawa et al., 1986; Wong and Goldstein, 1986) and it was found that the dispersion plays an important role and the different instability processes prefer different plasma $\beta$ regimes. While the decay instability is more characteristic to the low $\beta$ conditions, the modulational instability dominates under the high- $\beta$ conditions. For $\beta$ of the order of unity $(\beta \simeq 1)$ the beat instability plays also an important role (Hollweg, 1994).

For simplicity we assume the following scenario for the second stage of the foreshock wave evolution. The parent wave excited at the first stage by the right-hand resonant instability is subject to the decay instability, generating backward propagating waves (with respect to the parent wave direction) in the low $\beta$ regime, while it is subject to the modulational instability, generating forward propagating waves in the high $\beta$ regime (modulational instability). Therefore the cascade of the daughter waves results in both forward and backward propagating waves in the low $\beta$ regime, and only forward in the high $\beta$ regime.

\subsection{Cross helicity}

Examination on the above scenario is conveniently made by investigating the cross helicity density, defined as

$h_{c}=\boldsymbol{v} \cdot \frac{\boldsymbol{B}}{\sqrt{\mu_{0} \rho_{0}}}$,

where $\boldsymbol{v}, \boldsymbol{B}, \mu_{0}, \rho_{0}$ denote the flow velocity, the magnetic field, the permeability of free space, and the background mass density, respectively. The cross helicity density measures the correlation between the velocity and the magnetic field fluctuations, and its magnitude is maximized when the fluctuations are the Alfvénic, $\boldsymbol{v} \propto \pm \boldsymbol{B}$. We introduce the Elsässer variables

$z^{ \pm}=\boldsymbol{v} \pm \frac{\boldsymbol{B}}{\sqrt{\mu_{0} \rho_{0}}}$, 
in which case the cross helicity density is written in the form

$h_{c}=E^{+}-E^{-}$,

where $E^{ \pm}=\left|z^{ \pm}\right|^{2}$. This means that the cross helicity is essentially a measure of the energy difference between the two oppositely propagating Alfvén waves. The cross helicity density can be normalized to unity as

$\sigma_{c}=\frac{E^{+}-E^{-}}{E^{+}+E^{-}}$.

For example, $\sigma_{c}=1$ means the presence of the forward propagating (e.g. parallel to mean magnetic field) only, and vice versa. In the following we call $\sigma_{c}$ simply the cross helicity.

\subsection{Test for the hypothesis}

To test the hypothesis for the foreshock waves we assume the Alfvénic fluctuations and determine the cross helicity $\sigma_{c}$ using the four point magnetic field data in the foreshock region ahead of the terrestrial bow shock. The cross helicity is investigated under various conditions of $\beta$. We expect from the hypothesis that the cross helicity is dependent on the values of $\beta$. The diminished cross helicity for lower $\beta$ and the enhanced cross helicity for higher $\beta$, which results from the wave-wave interaction model at the second stage of the wave evolution.

In Sect. 2 we give brief introductions of the wave analysis methods: the dispersion relations, the wave number spectra, and the cross helicity. Those who are familiar with these methods may skip into Sect. 3 , where we present the statistics of the cross helicity and its dependence on $\beta$.

\section{Wave analysis}

\subsection{Wave telescope estimator}

The four point measurements enable one to determine the spectral density matrix as function of frequency $\omega$ and wave vector $\boldsymbol{k}$,

$\mathbf{E}(\omega, \boldsymbol{k})=\left(\begin{array}{lll}E_{x x} & E_{x y} & E_{x z} \\ E_{y x} & E_{y y} & E_{y z} \\ E_{z x} & E_{z y} & E_{z z}\end{array}\right)$.

While it is straightforward to determine the fluctuation amplitude at $\omega$ simply by Fourier transforming the time series data, the amplitude at $\boldsymbol{k}$ is not easily obtained by the Fourier transform procedure because of the limited number of the measurement points. It is, however, possible to determine the matrix $\mathbf{E}(\omega, \boldsymbol{k})$ when applying the so-called wave telescope estimator,

$\mathbf{E}(\omega, \boldsymbol{k})=\left[\mathbf{V}^{\dagger} \mathbf{H}^{\dagger} \mathbf{M}^{-1} \mathbf{H} \mathbf{V}\right]^{-1}$
Here the matrix $\mathbf{M}$ is the $12 \times 12$ cross spectral density matrix a function of frequency. It is determined by the measurements of the magnetic field fluctuations as

$\mathbf{M}(\omega)=\frac{1}{\Delta \omega}\left\langle\boldsymbol{B} \boldsymbol{B}^{\dagger}\right\rangle$,

where the symbol $\langle\cdot \cdot\rangle$ denotes the ensemble average and the dagger $\dagger$ means the Hermitian conjugate. $\mathbf{M}$ consists of the incorporated vector $\boldsymbol{B}$ for each component of the magnetic field fluctuations (the $\mathrm{x}-, \mathrm{y}-$, and $\mathrm{z}$-directions) and for each measurement point (spacecraft $1, \cdots, 4$ ),

$\boldsymbol{B}(\omega)=\left(\begin{array}{c}b_{1 x} \\ b_{1 y} \\ \vdots \\ b_{4 z}\end{array}\right)$.

The matrix $\mathbf{H}$ is called the steering matrix, as it steers the output matrix $\mathbf{E}$ for various wave vectors $\boldsymbol{k}$, and defined as

$\mathbf{H}(\boldsymbol{k})=\left(\begin{array}{l}\mathbf{I} e^{i \boldsymbol{k} \cdot \boldsymbol{r}_{1}} \\ \mathbf{I} e^{i \boldsymbol{k} \cdot \boldsymbol{r}_{2}} \\ \mathbf{I} e^{i \boldsymbol{k} \cdot \boldsymbol{r}_{3}} \\ \mathbf{I} e^{i \boldsymbol{k} \cdot \boldsymbol{r}_{4}}\end{array}\right)$,

where I denotes the $3 \times 3$ unit matrix, and $\boldsymbol{r}_{i}$ the position vector of the measurements. The matrix $\mathbf{V}$ imposes a further constraint on the spectral matrix, reflecting the fact that the magnetic field is divergence-free. It is defined as

$\mathbf{V}(\boldsymbol{k})=\mathbf{I}+\frac{\boldsymbol{k} \boldsymbol{k}^{\dagger}}{k^{2}}$,

where $k=|\boldsymbol{k}|$. Its algorithm was tested on simulated data to retrieve the input model. See, for example, Pinçon and Lefeuvre (1991); Motschmann et al. (1996) and Glassmeier et al. (2001). Derivation of Eq. (6) is shown in Appendix A.

We choose a coordinate system with the $\mathrm{z}$ axis aligned to the mean magnetic field direction. The $\mathrm{z}$ axis is parallel to the mean field when its sunward component is positive, and vice versa, so that the $\mathrm{z}$-axis is always oriented in the direction away from the bow shock. We use the Earth-to-sun direction projected into the plane perpendicular to the mean field as the $\mathrm{x}$-axis. Namely, our coordinate system is spanned by the following unit vectors

$\boldsymbol{e}_{x}=\boldsymbol{e}_{y} \times \boldsymbol{e}_{z}$

$\boldsymbol{e}_{y}=\boldsymbol{e}_{\mathrm{sun}} \times \boldsymbol{e}_{b}$

$\boldsymbol{e}_{z}=\frac{B_{x}}{\left|B_{x}\right|} \boldsymbol{e}_{b}$

where $\boldsymbol{e}_{b}$ and $\boldsymbol{e}_{\text {sun }}$ denote the unit vectors in the mean magnetic field direction and toward the sun, respectively. $B_{x}$ is the sunward component of the magnetic field, i.e. in the GSE$\mathrm{X}$ direction. 
Table 1. The ion beta, the Alfvén Mach number, the mean magnetic field strength, and the plasma density.

\begin{tabular}{lllll}
\hline Case & $\beta_{i}$ & $M_{A}$ & $B_{0}(\mathrm{nT})$ & $n_{0}\left(\mathrm{~cm}^{-3}\right)$ \\
\hline A & 0.29 & 5.05 & 6.64 & 5.88 \\
B & 0.22 & 4.19 & 10.23 & 6.05 \\
C & 2.02 & 4.83 & 8.02 & 1.98 \\
\hline
\end{tabular}

\subsection{Dispersion relations}

One of the useful applications of the wave telescope estimator is to determine the dispersion relations from the observations. We compute the total wave power $e(\omega, \boldsymbol{k})$ by taking trace of the matrix $\mathbf{E}$,

$e(\omega, \boldsymbol{k})=\operatorname{tr} \mathbf{E}$.

We investigate the total wave power $e$ at various frequencies (in the spacecraft frame of reference) and wave vectors, and identify the pairs of the frequency and the wave vector which yield peaks in the total wave power. It is worthwhile to note that the wave telescope estimator assumes the fluctuations as a set of incoherent wave fields, and therefore it is subject to interference, for example, when two waves possess exactly the same frequency. The frequencies can be transformed from the spacecraft frame frequency $\omega_{s c}$ to the plasma rest frame frequency $\omega_{r e}$ (co-moving with the plasma bulk flow) using the Doppler relation $\omega_{r e}=\omega_{s c}-\boldsymbol{k} \cdot \boldsymbol{V}_{0}$, when $\boldsymbol{k}$ is determined by the wave telescope method and $\boldsymbol{V}_{0}$ (the mean flow velocity vector) is known.

We apply the dispersion analysis to the measurements provided by the four Cluster spacecraft (Escoubet et al., 2001). The magnetic field measurements of the FGM instrument (Balogh et al., 2001) are used to determine the pairs of the frequency and the wave vector, and the ion measurements of the CIS-HIA instrument (Rème et al., 2001) are used to determine the mean flow velocity. Various kinds of curves of the dispersion relations are identified in the foreshock region by this analysis. Figure 1 displays three distinct cases of the dispersion curves for the field-aligned wave numbers, $\omega_{r e}\left(k_{z}\right)$. Hereafter $\omega_{r e}$ is normalized to the proton cyclotron frequency $\Omega_{p}$, and $k_{z}$ is normalized to the ion inertial wave number $\Omega_{p} / V_{A}$. The Alfvén speed is determined by the magnetic field and the ion measurements for each time interval. Table 1 summarizes the plasma and magnetic field parameters for the three cases: the ion beta $\beta_{i}$ (assuming protons); the Alfvén Mach number $M_{A}$; the mean magnetic field strength $B_{0}$; and the plasma density $n_{0}$.

In the case A (16 February 2002, 07:00-07:30 UT) almost all of the waves are identified in the direction away from the shock. Only few waves propagate in the opposite direction at very low frequencies $\left(\omega_{r e} \simeq 0\right)$. The dispersion branch starts at $\left(\omega_{r e}, k_{z}\right)=(0,0)$ and extends solely in the anti-parallel di- rection, keeping the phase speed almost at the Alfvén speed $v_{A}$ at low frequencies, $\omega_{r e} / k_{z} \simeq v_{A}$. At $\omega_{r e} \sim 0.5$ the dispersion branch starts to be bent and deviates from the linear branch toward higher frequencies. This is characteristic to the low frequency part of the magnetosonic/whistler waves.

The case B (27 April 2002, 02:00-02:30 UT) exhibits waves that are counter-propagating at low frequencies. Most of the identified waves propagate in the $+z$ direction at various frequencies $\left(\omega_{r e} \leq 3\right)$, but some waves propagate in the opposite direction at low frequencies $\left(\omega_{r e} \leq 0.4\right)$. The dispersion branch in the $+z$ direction, can be approximated by a straight line (linear dispersion relation), but the phase speed does not agree with the Alfvén speed $\left(\omega_{r e} \geq k_{z} v_{A}\right)$. In the $-z$ direction it is not clear if the waves follow any dispersion relation, since only few waves are identified.

The case C (6 March 2002, 00:30-01:00 UT) exhibits an example of the enhanced counter-propagating waves. Compared to the case $\mathrm{B}$, the identified waves look rather scattered in the dispersion diagram. One branch starts at $\left(\omega_{r e}, k_{z}\right) \simeq(0,0)$ and extends in the $+z$ direction to $\left(\omega_{\text {re }}, k_{z}\right)=(3,-1.5)$, while the branch in the $-z$ direction stops at $\omega_{r e} \simeq 1.5$. The scatter in the dispersion diagram makes it difficult to identify the dispersion relation, but roughly speaking, the waves follow the magnetosonic/whistler branch.

\subsection{Wave number spectra}

The second application of the wave telescope estimator is the wave number spectra (energy spectra in the wave number domain). We determine two kinds of wave number spectra: $E^{+}\left(k_{z}\right)$ and $E^{-}\left(k_{z}\right)$. They represent the energy for the Alfvénic fluctuations propagating along the magnetic field away from the shock $\left(E^{+}\right)$, and toward the shock $\left(E^{-}\right)$, respectively, and are determined as follows.

$$
\begin{aligned}
& E^{+}\left(k_{z}\right)=\frac{\alpha}{\Delta k} \int d \omega_{r e}\left[E_{x x}\left(\omega_{r e}, k_{z}\right)+E_{y y}\left(\omega_{r e}, k_{z}\right)\right] \\
& E^{-}\left(k_{z}\right)=\frac{\alpha}{\Delta k} \int d \omega_{r e}\left[E_{x x}\left(\omega_{r e},-k_{z}\right)+E_{y y}\left(\omega_{r e},-k_{z}\right)\right],
\end{aligned}
$$

where the integration is made over the frequency in the plasma rest frame.

In the wave telescope procedure each elements of the matrix $E_{i j}(\omega, \boldsymbol{k})$ is given in unit of $\mathrm{nT}^{2} / \mathrm{Hz}$, which originates in the cross spectral density matrix $\mathbf{M}$ in the frequency domain. The integration in the above equations provides the spectra in units of the squared amplitude $\left(\mathrm{nT}^{2}\right)$, and then it is divided by $\Delta k$ to transform the unit from the squared amplitude to $\mathrm{nT}^{2} \mathrm{~km}$. We use an equidistant grid in the logarithmic scale of the wave number, which results in a scaling law $\Delta k \propto k$. The factor $\alpha$ scales the wave number spectrum such that it yields the variance (which is the mean squared amplitude) of the magnetic field fluctuations when integrated over the wave number domain. We use the magnetic field variance 
(A) 16 Feb, 2002, 0700-0730 UT

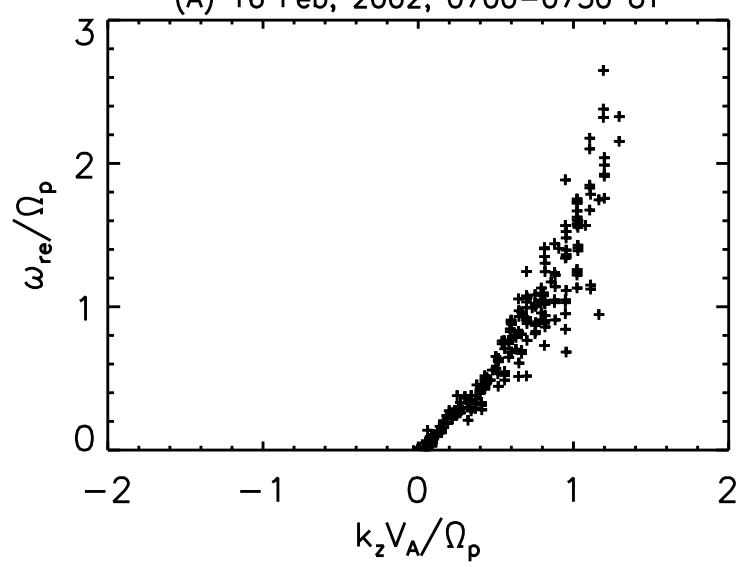

(B) $27 \mathrm{Apr}, 2002,0200-0230$ UT

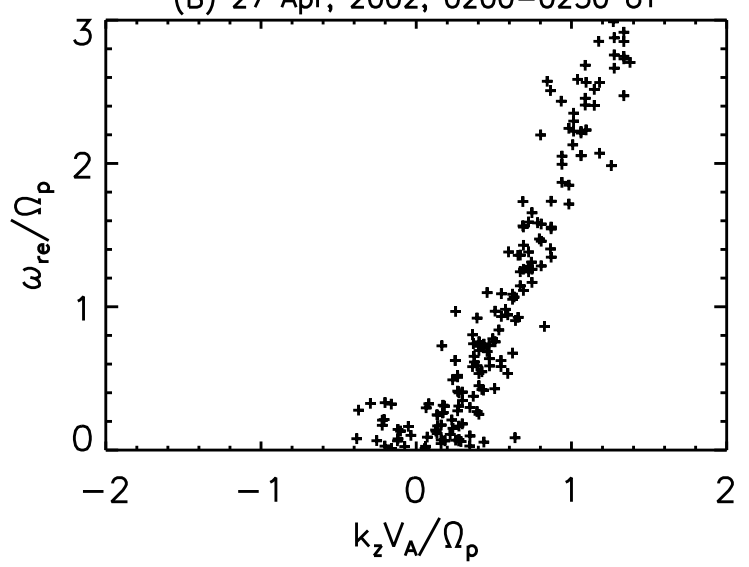

(C) 06 Mar, 2002, 0030-0100 UT

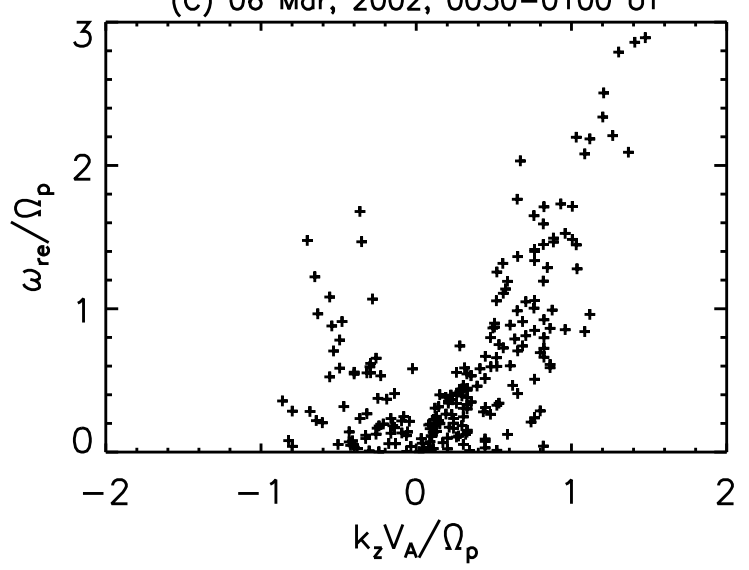

Fig. 1. Wave frequencies in the plasma rest frame and wave numbers aligned to the mean magnetic field. The $+z$ direction is away from the shock toward upstream.

in the time domain for the normalization, assuming that the fluctuations are homogeneous.

The wave number spectra for the three cases A, B, and $\mathrm{C}$ are displayed in Fig. 2. The wave numbers are scaled

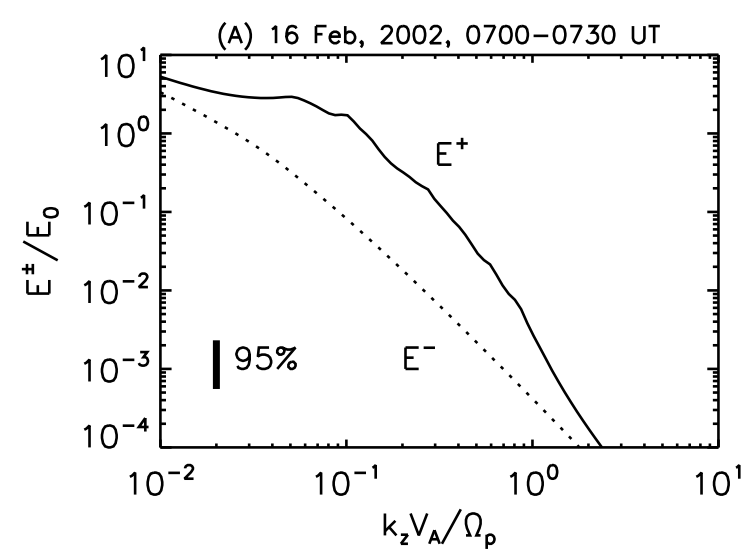

(B) $27 \mathrm{Apr}, 2002,0200-0230$ UT
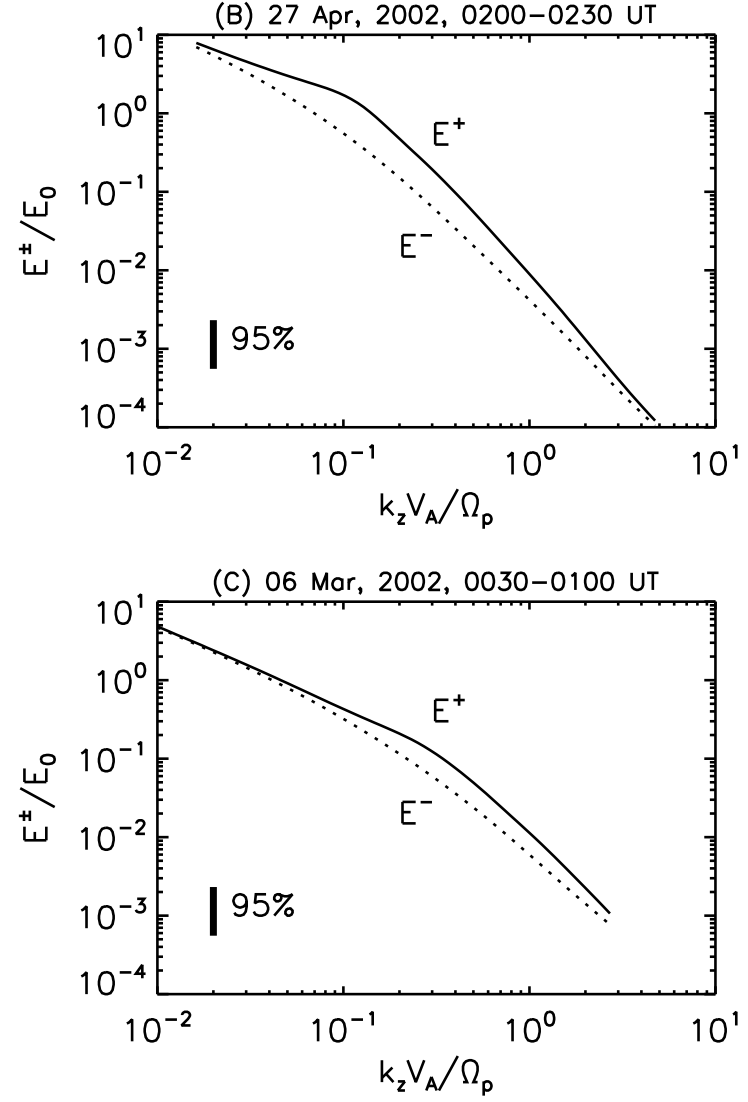

Fig. 2. Energy spectra of the Alfvénic fluctuations in the wave number domain for the wave number away from the shock (solid curve) and for the wave number toward the shock (dotted curve).

to the ion inertial wave number and the energy spectra are scaled to $E_{0}=B_{0}^{2} V_{A} / \Omega_{p}$ (the squared mean magnetic field strength divided by the ion inertial wave number). Qualitatively the three wave number spectra exhibit common features, while they are quantitatively different from one another. There are two characteristics that are common. One is that $E^{+}$(solid line) is always greater than $E^{-}$(dotted 

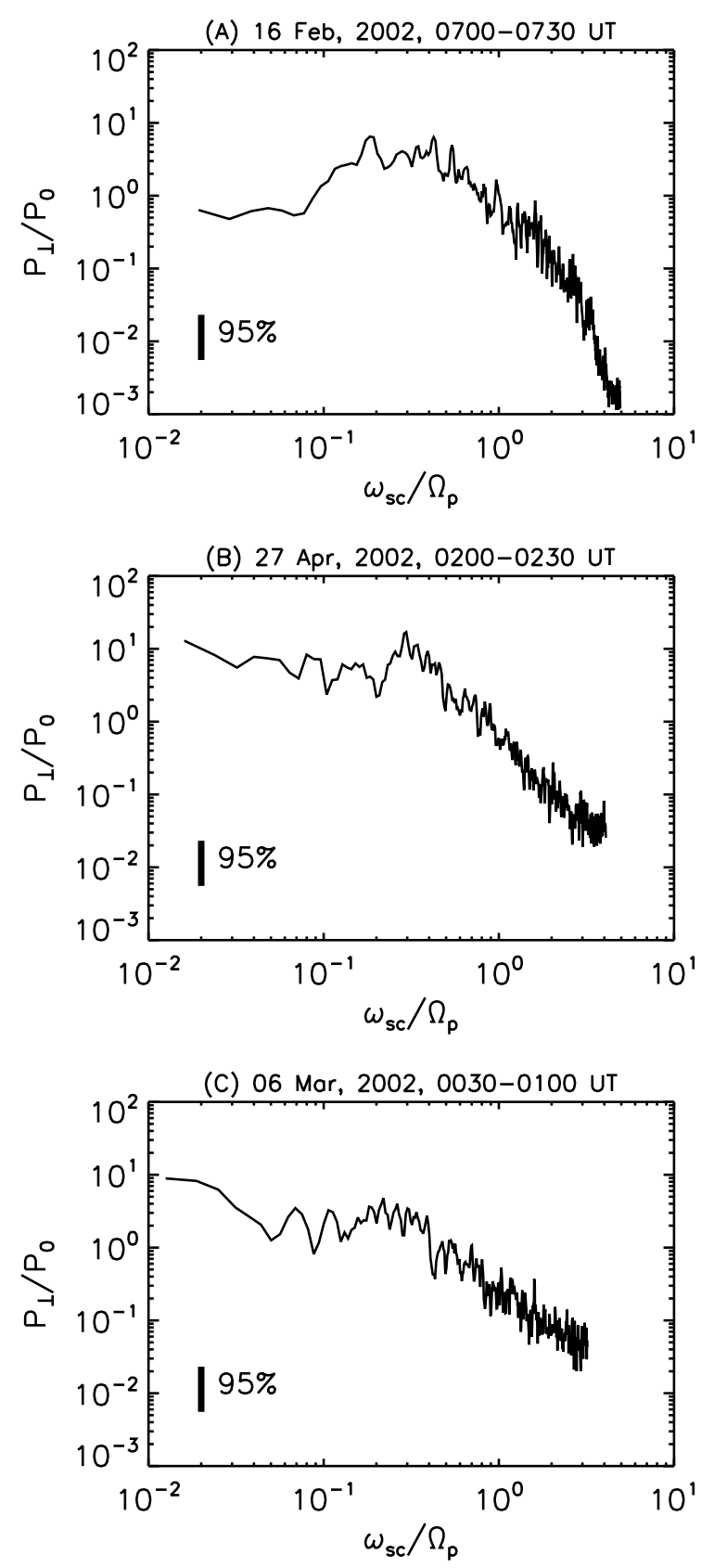

Fig. 3. Energy spectra of the magnetic field fluctuations perpendicular to the mean field in the frequency domain.

line), that is the foreshock fluctuations contain more energy in the waves propagating away from the shock than that for the waves toward the shock. Another point is that the spectra exhibit humps which are an indication of the energy injection. The humps can be seen only in $E^{+}$, while $E^{-}$shows only monotonously decaying curves toward the larger wave numbers. The injection scale is, however, slightly different from case to case. It becomes shifted gradually toward the larger wave numbers from the case A to the case C. A closer inspection yields the values of the normalized wave numbers for the injection scales: $k_{z} \approx 0.05-0.1$ (case A); $0.1-0.2$ (case B); and 0.3 (case C). The difference between $E^{+}$and $E^{-}$becomes also smaller from the case A to the case C.

\section{Frequency spectra}

For comparison, frequency spectra for the three events are displayed in Fig. 3, where the wave power for the magnetic field fluctuation perpendicular to the mean field $P_{\perp}$ is scaled using $P_{0}=2 \pi B_{0}^{2} V_{A} / V_{0} \Omega_{p}$ and the spacecraft frame frequency is scaled to the proton cyclotron frequency. It is important to note that one cannot distinguish between $E^{+}$ and $E^{-}$from the magnetic field measurements only in the frequency spectra. Of course, the frequency spectra can be determined using the Elsässer variables, but still one cannot reach smaller scales due to the limited sampling rate for the particle measurements, which is typically the time scale of the spacecraft spin, a few seconds. The frequency spectra exhibit more or less resemblance to the wave number spectra. But this is not surprising, as the solar wind streams at the supersonic and super-Alfvénic speed and the spatial structures are recorded as the temporal variations by the spacecraft. However, the frequency spectra exhibit spikes on various scales. Some are small scale, sharp spikes and some are large scale, blunt spectral hump. Generally speaking, it is difficult to identify the energy injection scale and the powerlaw decay of the spectra unambiguously.

\subsection{Dimensionless cross helicity}

The third application of the wave telescope estimator is the dimensionless cross helicity $\sigma_{c}$. We apply the two energy spectra $E^{+}$and $E^{-}$as determined in Eqs. (15) and (16) to the Eq. (4). In our coordinate system $E^{+}$denotes the waves propagating away from the shock, hence $\sigma_{c}=1$ means that all the Alfvénic fluctuation energy represents waves propagating in this direction.

The cross helicity for the three cases are displayed in Fig. 4, which reinterprets the difference between the two spectra $E^{+}$and $E^{-}$in Fig. 3. The cross helicity is positive at various wave numbers in all three cases. The case A exhibits an increase of the cross helicity from 0.2 to 0.9 toward the wave number $k_{z} \approx 0.1$. The largest values is kept at $\sigma_{c} \approx 0.9$ for the wave numbers $0.1<k_{z}<1$, and falls into $\sigma_{c} \approx 0.3$ on the smaller scales $\left(k_{z}>1\right)$. The case B exhibits a moderate peak in the cross helicity at $\sigma_{c} \approx 0.5$ at the wave number $k_{z} \approx 0.1$, and the case $\mathrm{C}$ exhibits an even more moderate peak at $\sigma_{c} \approx 0.4$ at the wave number $k_{z} \approx 0.3$. It is interesting to note that the information in the dispersion curves displayed in Fig. 1 is also stored in the cross helicity. The more counter-propagating waves are present in the $-z$ direction in the dispersion diagram, the smaller the cross helicity becomes. The wave numbers yielding the peaks in the cross 
helicity also agrees with the energy injection scale obtained in the previous section.

\section{Statistical study}

\subsection{Event selection}

The dimensionless cross helicity $\sigma_{c}$ is investigated for various time intervals of the observations in the foreshock region. Following criteria are imposed to select the time intervals for the foreshock wave observations: (1) mission phase with the smallest inter-spacecraft distance, $100 \mathrm{~km}$, to resolve as smallest wavelengths as possible (February to May 2002), (2) existence of supra-thermal ion populations, namely backstreaming ions in addition to the solar wind ion population, (3) enhanced level of magnetic field fluctuations. We select the time intervals with the fluctuating energy more than $30 \%$ of the mean field energy,

$\left\langle\frac{\left|\delta B_{x}\right|^{2}+\left|\delta B_{y}\right|^{2}+\left|\delta B_{z}\right|^{2}}{B_{0}^{2}}\right\rangle^{1 / 2} \geq 0.3$

where $\delta B_{x}, \delta B_{y}$, and $\delta B_{z}$ denote the three components of the fluctuating magnetic field, and $B_{0}$ denotes the mean magnetic field strength. The mean field is defined by averaging the field strength $\boldsymbol{B}_{0}=\langle\boldsymbol{B}\rangle$ so that $\boldsymbol{B}_{0}$ can be treated as a uniform constant vector for each $30 \mathrm{~min}$ interval. The ion measurements of the CIS-HIA instrument are used in step (2). As a result we obtain 32 intervals for the statistical study.

\subsection{Distribution of cross helicity}

Figure 5 displays the superposed cross helicity for all the events. As the three case studies show in the previous section, the cross helicity is positive at various wave numbers. Statistically the foreshock is indeed dominated by the waves propagating away from the shock. On the largest scale $\left(k_{z} \sim 0.01\right.$, where $k_{z}$ is normalized to the ion inertial scale) the cross helicity approaches to zero. At the wave numbers $k_{z} \sim 0.1$ the cross helicity reaches its maximum, but the distribution of the maximum cross helicity ranges from almost zero to almost unity. On the smaller scales $\left(k_{z}>1\right)$ the cross helicity is diminished. We estimate the injection scale of the cross helicity for the wave numbers yielding the maximum cross helicity. Figure 6 displays the histogram of the peak wave numbers on the logarithmic scale, and the distribution peaks at about $\log _{10}\left(k_{z}\right)=-1$, namely $k_{z}=0.1$. This value agrees well with the typical wave numbers for the maximum growth rate of the right-hand resonant instability (Gary, 1993) and also justifies the first part of our hypothesis. The reason for the decrease of the cross helicity on the smaller scales is not clear. It may mean the dissipation of the forward propagating waves or it may come from the wave-wave interactions.
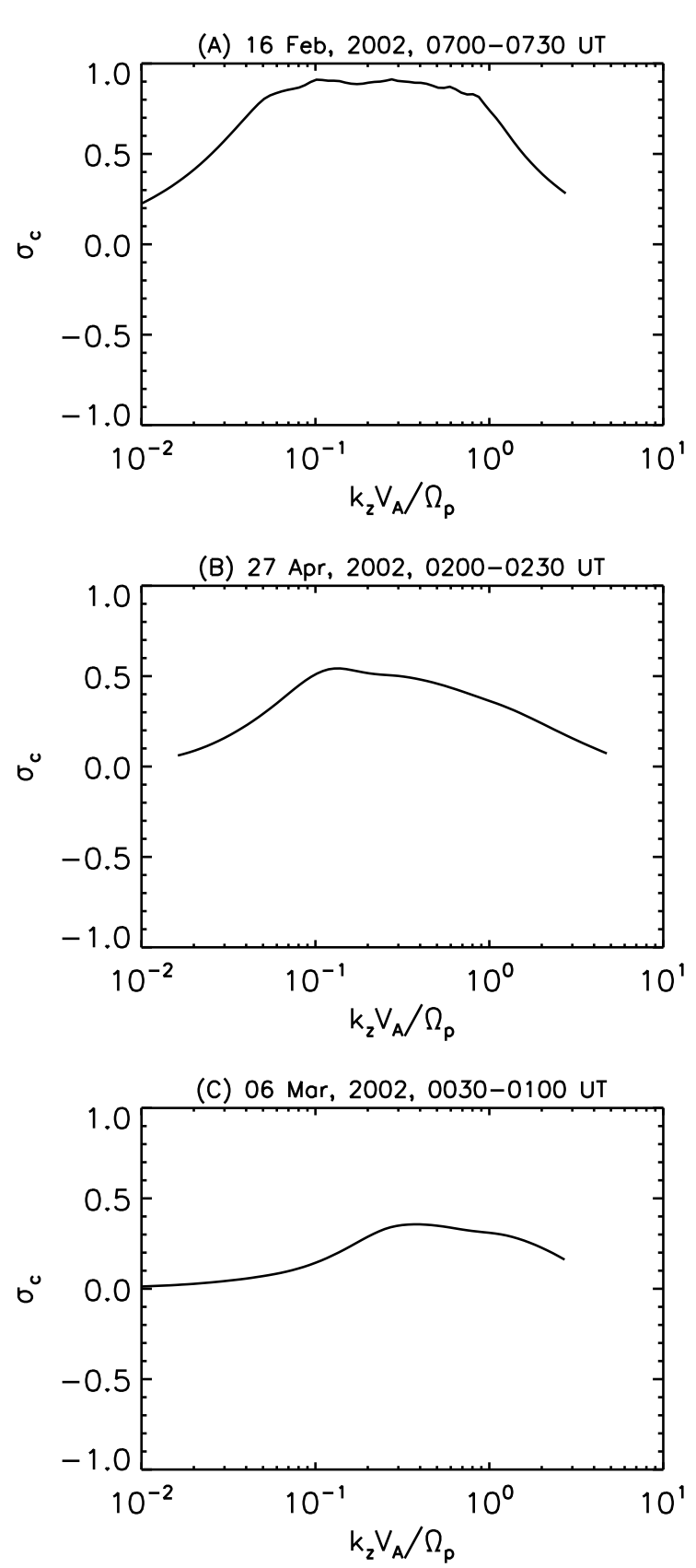

Fig. 4. Dimensionless cross helicity in the wave number domain. Positive cross helicity means dominance of Alfvén waves propagating away from the shock.

\subsection{Relation to $\beta$}

Finally we are ready to study the relation between the cross helicity and the plasma parameter $\beta$. We use the density and the temperature data obtained by the ion instrument (CISHIA) to determine the ion beta, $\beta_{i}$. We investigate three kinds of the cross helicity under various conditions of the 


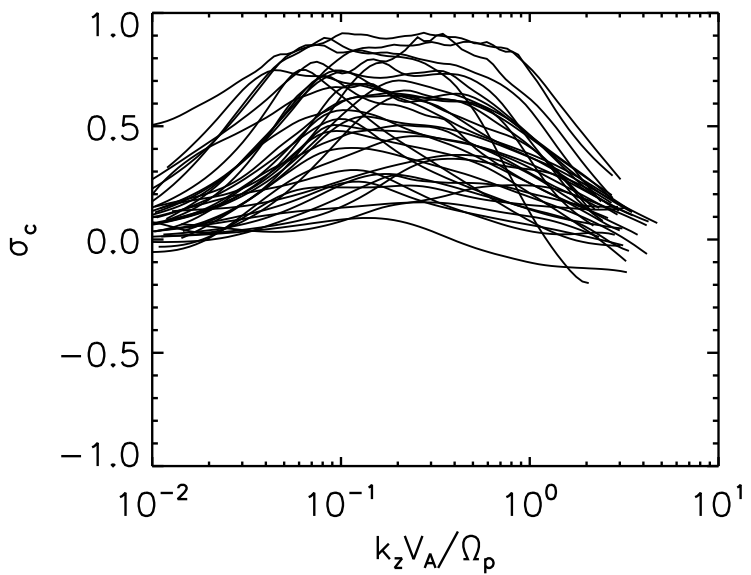

Fig. 5. Superposed plot of dimensionless cross helicity in the wave number domain.

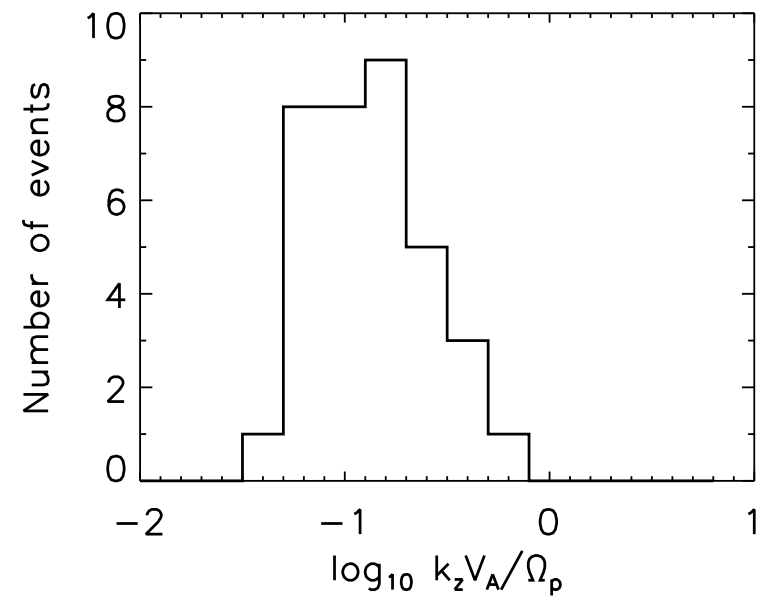

Fig. 6. Histogram of wave numbers corresponding to maximum cross helicity.

$\beta_{i}$ : the maximum cross helicity, the large scale mean, and the small scale mean. Concluding the result first of all, none of them exhibit a clear relation to $\beta_{i}$. The maximum cross helicity varies from 0.1 to 0.9 , while $\beta_{i}$ varies from 0.3 to 6 . The maximum cross helicity is distributed relatively uniformly over $\beta_{i}$ (Fig. 7). When averaged over the larger scales $\left(0.01<k_{z}<0.1\right)$, the cross helicity exhibits the values between 0 and 0.5 , and it is distributed again relatively uniformly over $\beta_{i}$ (Fig. 8a). The cross helicity averaged over the smaller scales $\left(k_{z}>1\right)$ also displays a relatively flat distribution over $\beta_{i}$, though the cross helicity is more concentrated around $\sigma_{c} \approx 0.2$ (Fig. 8b).

For comparison, Fig. 9 displays schematically the regimes of the three instabilities (the right-hand resonant instability, the decay and the modulational instabilities) expected in our hypothesis. The ion/ion right-hand resonant instability is a driver of the primary waves and we expect its presence at

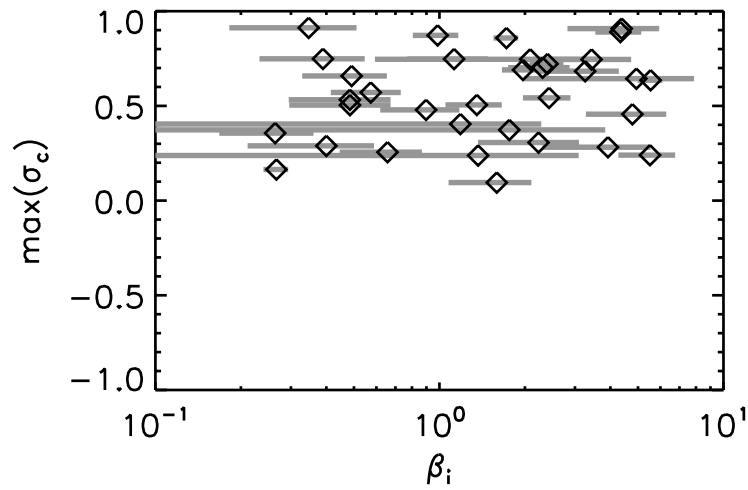

Fig. 7. Maximum cross helicity plotted versus $\beta_{i}$. The horizontal bars in gray represent the error of $\beta_{i}$ (standard deviation).

various values of $\beta$. On the other hand, the decay and the modulational instabilities are dependent on $\beta$. The decay instability prefers the low $\beta$ condition, and produces counterpropagating waves (backward to the parent wave direction), which reduces the cross helicity. The modulational instability produces prefers the high $\beta$ condition and produces waves propagating only in the forward direction. The sketch shows the regime of the right-hand resonant instability at the enhanced cross helicity $\left(\sigma_{c} \sim 1\right)$ regardless of the magnitude of $\beta$. The sketch also exhibit the decay instability at the reduced cross helicity $\left(\sigma_{c} \sim 0\right)$ and low $\beta$ (smaller than unity), and the modulational instability at enhanced cross elicits and high $\beta$ (larger than unity).

It is true that some events shown in Figs. 7, 8a, and $8 \mathrm{~b}$ agree with the expectations, for example, the distribution of the maximum cross helicity (Fig. 7) qualitatively overlaps with the regime for the right-hand resonant instability, but some of the events deviate from the expectation for the decay and the modulational instabilities. The hypothesis with the decay and the modulational instabilities seems to fail in our study.

\section{Discussion}

\subsection{Right-hand resonant instability}

The result that the maximum cross helicity is always on the positive side suggests that the primary waves propagate in the direction away from the shock. This excludes the ion/ion non-resonant mode for the most likely source of the foreshock waves, since this mode excites the waves in the opposite direction to the ion beam (i.e. toward the shock). Furthermore, the dispersion relations in Fig. 1 prefers the right-hand mode, as the dispersion branch is connected beyond the ion cyclotron frequency and the curves of the dispersion relation are reminiscent of the magnetosonic/whistler mode. This justifies the first stage of our hypothesis that the right-hand 


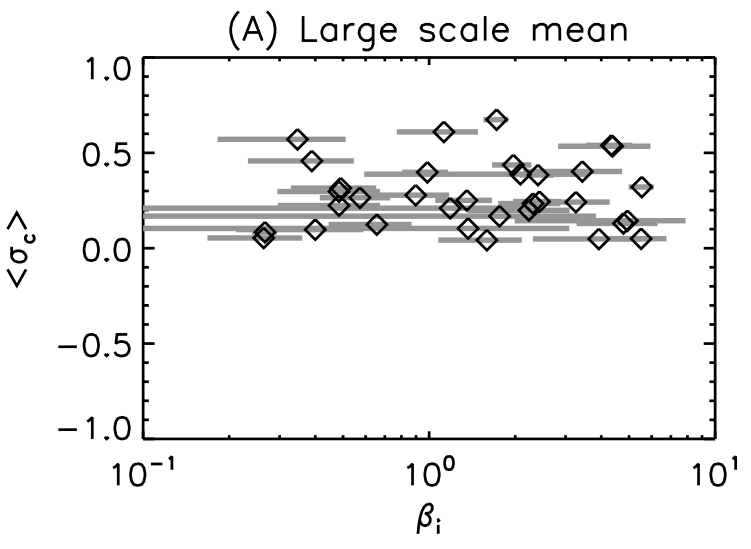

(B) Small scale mean

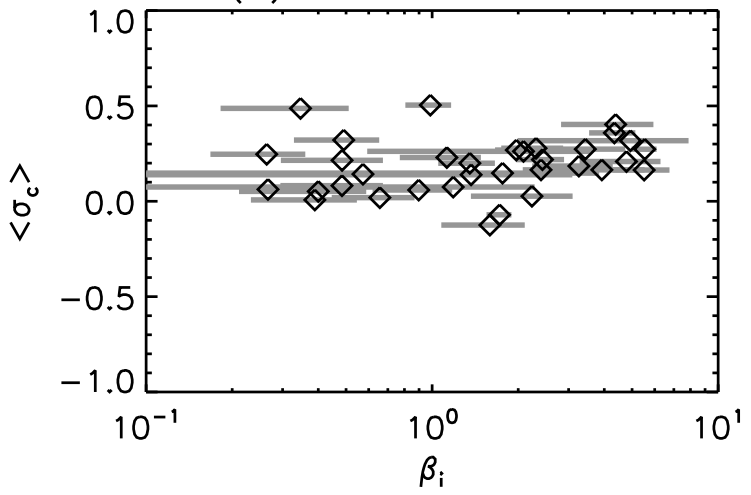

Fig. 8. Cross helicity averaged over the large scales $\left(k_{z} V_{A} / \Omega_{p}<0.1\right.$, top panel) and over the small scales $\left(k_{z} V_{A} / \Omega_{p}>1\right.$, bottom panel).

resonant instability is the most likely source for the primary waves.

\subsection{Parametric instabilities}

It is interesting that the cross helicity is almost arbitrary between 0 and 1 at various values of $\beta_{i}$. This cannot be explained by a simple picture of the parametric instabilities as proposed in the hypothesis. Spangler et al. (1997) argue on the basis of the frequency spectra that the evidence for the decay instability was only rarely present, but be present when the theoretical growth rate is high. Therefore it is not decided yet if the parametric instability really occurs in the foreshock, but here we summarize possible explanations about the reason why the parametric instability model fails.

(1) The $\beta$-dependence is altered if kinetic effects are included (Mjølhus and Wyller, 1988; Spangler, 1989; Vasquez, 1995; Araneda, 1998; Bugnon et al., 2004; Nariyuki and Hada, 2006b). For example, Vasquez (1995) argues that the right-hand polarized wave is also subject to the decay instability even in the high- $\beta$ regime. (2) The existence of broadband waves or inhomogeneity of the background medium

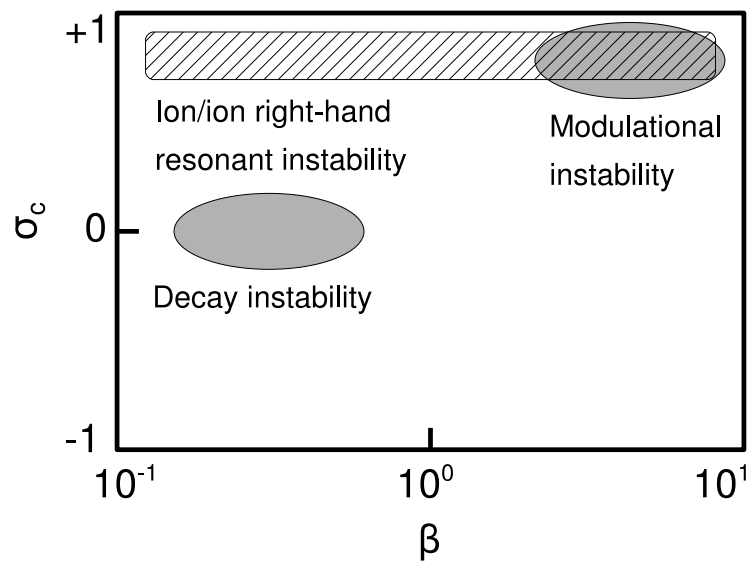

Fig. 9. Regimes of three instabilities expected in the hypothesis.

may be important as well. A different kind of instability process may exist when the parent wave is not monochromatic but a mixture of side-band waves (Nariyuki and Hada, 2007). (3) While the models of the parametric instabilities are usually discussed in the one-dimensional context (e.g. variations only in the $\mathrm{x}$-direction), the waves may be unstable in the $\mathrm{y}$-, or z-directions like wave refraction (Mjølhus and Hada, 1990; Vinaz and Goldstein, 1991; Laveder et al., 2002). (4) In the present paper we used only the ion measurements for estimating $\beta$. The ratio of the electron to the proton temperature is, however, typically between 1 and 4 in the solar wind (Newbury et al., 1998), thus inclusion of the electron temperature effect could increases the estimated values of $\beta$. (5) Steepened waves or even discontinuities such as shocklets may have been decomposed into forward and backward waves. In this picture the number of the steepened wave is more important than the the parametric instabilities. (6) Furthermore, the waves that already exist in the solar wind should be taken into account. For example, the Alfvén waves coming from the sun may have been counted in our study, which results in a smaller value of the cross helicity. From this point of view, the foreshock observations should be further classified into the fast and the slow solar wind intervals.

Our study is based on the second order moments such as the energy and the cross helicity. It is worthwhile to note that the direct evidence of the parametric instabilities can be achieved only by determining higher order moments, e.g. 3 wave field correlation at different frequencies and wave numbers is able to examine the three wave interaction process (Dudok de Wit et al., 1999). This kind of analysis method is currently developed to apply to the multi-point measurements.

Also, one needs to identify on what scales the dissipation effect is operating, as both the backward propagating waves and the dissipation of the forward propagating waves tend to reduce the cross helicity, For this purpose, careful 
investigations of the energy spectra both in the frequency and in the wave number domain would be of some help. Thanks to the recent instrumentation providing high time resolution in the field measurements, the dissipation of the energy should be visible in the frequency spectra, but one cannot obtain the exact spatial scales. But combining the frequency and the wave number spectra would enable one to determine the dissipation scales.

\section{Conclusions}

We have used data analysis from 32 Cluster crossings of the foreshock to show that there are a variety of wave populations in the foreshock. Some propagate in the direction of the ion beam away from the shock, and some propagate in the opposite direction. Also some waves follow the dispersion branch for the magnetosonic/whistler mode, and some deviate from the dispersion branch. The first stage of the foreshock wave evolution is realized by the ion/ion right-hand resonant instability. The ion beams originating in the specular particle reflection are indeed the ultimate source for the foreshock wave. However, it is not clear what really happens at the second stage. The origin of of the backward propagating waves is still an unsolved problem. Nevertheless, The higher order statistics will be a powerful tool to identify the wave-wave interactions, where the frequency and the wave number resonance conditions can be directly investigated.

\section{Appendix A}

\section{Derivation of Eq. (6)}

We consider a constrained optimization problem minimizing the cost matrix

$$
\mathbf{C}=\mathbf{W}^{\dagger} \mathbf{M W}+\lambda\left(\mathbf{W}^{\dagger} \mathbf{H}-\mathbf{I}\right) .
$$

The first term on the right-hand side denotes the cross spectral density matrix $\mathbf{E}$ in the frequency and the wave number domain, and the second term denotes a constraint. $\mathbf{M}$ is the cross spectral density matrix for all the measured variables at frequency $\omega$

$$
\mathbf{M}(\omega)=\frac{1}{\Delta \omega}\left(\begin{array}{cccc}
\left\langle b_{1 x} b_{1 x}^{*}\right\rangle & \left\langle b_{1 x} b_{1 y}^{*}\right\rangle & \cdots & \left\langle b_{1 x} b_{4 z}^{*}\right\rangle \\
\left\langle b_{1 y} b_{1 x}^{*}\right\rangle & \left\langle b_{1 y} b_{1 y}^{*}\right\rangle & & \\
\vdots & & \ddots & \\
\left\langle b_{4 z} b_{1 x}^{*}\right\rangle & & & \left\langle b_{4 z} b_{4 z}^{*}\right\rangle
\end{array}\right)
$$

$\mathbf{W}$ is the weight matrix subject to

$$
\mathbf{W}^{\dagger} \mathbf{H}=\mathbf{I}
$$

See Eq. (9) for the definition of $\mathbf{H}$ (the steering matrix). This constraint imposes a unit gain of the wave amplitude for every pair of the frequency and the wave number, while the weights are chosen to minimize the output from all other portions of the spectrum. $\lambda$ is the Lagrangian multiplier $\lambda$.

This optimization problem can be solved analytically, yielding the weight matrix

$\mathbf{W}(\omega, \boldsymbol{k})=\frac{\mathbf{M}^{-1} \mathbf{H}}{\mathbf{H}^{\dagger} \mathbf{M}^{-1} \mathbf{H}}$.

Therefore $\mathbf{E}$ is given as

$$
\begin{aligned}
\mathbf{E}(\omega, \boldsymbol{k}) & =\mathbf{W}^{\dagger} \mathbf{M W} \\
& =\left[\mathbf{H}^{\dagger} \mathbf{M}^{-1} \mathbf{H}\right]^{-1}
\end{aligned}
$$

In addition, we put the second constraint for the divergencefree magnetic field, which results in the replacement

$\mathbf{H} \rightarrow \mathbf{H V}$,

where $\mathbf{V}$ is defined in Eq. (10). This gives the estimator for the cross spectral density matrix as presented in Eq. (6).

Acknowledgements. This work is financially supported by the Federal Ministry for Education and Research (Bundesministerium für Bildung und Forschung) and the German Aerospace Center (Deutsches Zentrum für Luft- und Raumfahrt) under contract 50OC0103. YN thanks H. Rème and I. Dandouras for providing the CIS-HIA data, M. Hoshino and all the participants at the NLW6 meeting in Fukuoka, Japan.

Edited by: A. C. L. Chian

Reviewed by: S. P. Gary and S. Spangler

\section{References}

Araneda, J.: Parametric instabilities of parallel propagating Alfvén waves: Kinetic effects in the MHD-model, Phys. Scr. T75, 164 168, 1998.

Balogh, A., Carr, C. M. , Acuña, M. H., Dunlop, M. W., Beek, T. J., Brown, P., Fornaçon, K.-H., Georgescu, E., Glassmeier, K.H., Harris, J., Musmann, G., Oddy, T., and Schwingenschuh, K.: The Cluster magnetic field investigation: overview of in-flight performance and initial results, Ann. Geophys., 19, 1207-1217, 2001, http://www.ann-geophys.net/19/1207/2001/.

Bugnon, G., Passot, T., and Sulem, P. L.: Landau-fluid simulations of Alfvén-wave instabilities in a warm collisionless plasma, Nonlin. Processes Geophys., 11, 609-618, 2004, http://www.nonlin-processes-geophys.net/11/609/2004/.

Derby Jr., N. F.: Modulational instability of finite-amplitude, circularly polarized Alfvén waves, Astrophys. J., 224, 1013-1016, 1978.

Dudok de Wit, T., Krasnosel'skikh, V. V., Dunlop, M., and Lühr, H.: Identifying nonlinear wave interactions in plasmas using twopoint measurements: A case study of Short Large Amplitude Magnetic Structures (SLAMS), J. Geophys. Res. 104, 17079 17 090, 1999.

Escoubet, C. P., Fehringer, M., and Goldstein, M. L.: The Cluster mission, Ann. Geophys., 19, 1197-1200, 2001, http://www.ann-geophys.net/19/1197/2001/. 
Galeev, A. A., Oraevskii, V. N., and Sagdeev, R. Z.: Universal instability of an inhomogeneous plasma in a magnetic field, Sov. Phys. JETP, 17, 615-620, 1963.

Gary, S. P.: Theory of Space Plasma Microinstability, Cambridge: Cambridge University Press, 1993.

Glassmeier, K.-H., Motschmann, U., Dunlop, M., Balogh, A., Acuña, M. H., Carr, C., Musmann, G., Fornaçon, K.-H., Schweda, K., Vogt, J., Georgescu, E., and Buchert, S.: Cluster as a wave telescope - first results from the fluxgate magnetometer, Ann. Geophys., 19, 1439-1447, 2001 (Corrigendum 21, 1071, 2003).

Goldstein, M. L.: An instability of finite amplitude circularly polarized Alfvén waves, Astrophys. J., 219, 700-704, 1978.

Hollweg, J, V.: Modulational and decay instability of a circularly polarized Alfvén wave, J. Geophys. Res., 99(23), 431-447, 1994.

Laveder, D., Passot, T., and Sulem, P. L.: Transverse dynamics of dispersive Alfvén waves, I. Direct numerical evidence of filamentation, Phys. Plasmas, 9, 293-304, 2002.

Longtin, M. and Sonnerup, B.: Modulational instability of circularly polarized Alfvén waves, J. Geophys. Res., 91, 798-801, 1986.

Mio, K., Ogino, T., Minami, K., and Takeda, S.: Modulational instability and envelope-solitons for nonlinear Alfvén waves propagating along the magnetic field in plasmas, J. Phys. Soc. Japan, 41, 667-673, 1976.

Mjølhus, E.: On the modulational instability of hydromagnetic waves parallel to the magnetic field, J. Plasma Phys., 16, 321334, 1976.

Mjølhus, E. and Wyller, J.: Nonlinear Alfvén waves in a finite beta plasma, J. Plasma Phys., 40, 299-318, 1988.

Mjølhus, E. and Hada, T.: Oblique stability of circularly polarized MHD waves, J. Plasma Phys., 43, 257-268, 1990.

Motschmann, U., Woodward, T. I., Glassmeier, K.-H., and Pinçon, J. L.: Wavelength and direction filtering by magnetic measurements at satellite arrays: Generalized minimum variance analysis, J. Geophys. Res., 101, 4961-4965, 1996.

Narita, Y., Glassmeier, K.-H., Schäfer, S., Motschmann, U., Sauer, K., Dandouras, I., Fornaçon, K.-H., Georgescu, E., and Rème, H.: Dispersion analysis of ULF waves in the foreshock using cluster data and the wave telescope technique, Geophys. Res. Lett., 30, SSC 43-1, doi:10.1029/2003GL017432, 2003.

Narita, Y. and Glassmeier, K.-H.: Dispersion analysis of lowfrequency waves through the terrestrial bow shock, J. Geophys. Res., 110, A12215, doi:10.1029/2005JA011256, 2005.

Narita, Y., Glassmeier, K.-H., and Treumann, R. A.: Wave-number spectra and intermittency in the terrestrial foreshock region, Phys. Rev. Lett.,97(19), 191 101, doi:10.1103/PhysRevLett.97.191101, 2006.
Nariyuki, Y. and Hada, T.: Remarks on nonlinear relation among phases and frequencies in modulational instabilities of parallel propagating Alfvén waves, Nonlin. Processes Geophys., 13, 425-441, 2006a.

Nariyuki, Y. and Hada, T.: Kinetically modified parametric instabilities of parallel propagating Alfvén waves: ion kinetic effects, Phys. Plasmas, 13, 124 501, doi:10.1063/1.2399468, 2006b.

Nariyuki, Y. and Hada, T.: Magnetohydrodynamic parametric instabilities of parallel propagating incoherent Alfvén waves, Earth Planets Space, 59, e13-e17, 2007.

Newbury, J. A., Russell, C. T., Phillips, J. L., and Gary, S. P.: Electron temperatures in the ambient solar wind: Typical properties and a lower bound at $1 \mathrm{AU}, \mathrm{J}$. Geophys. Res., 103, 9553-9566, 1998.

Pinçon, J. L. and Lefeuvre, F.: Local characterization of homogeneous turbulence in a space plasma from simultaneous measurement of field components at several points in space, J. Geophys. Res., 96, 1789-1802, 1991.

Rème, H., Aoustin, C., Bosqued, J. M., Dandouras, I., et al.: First multispacecraft ion measurements in and near the Earth's magnetosphere with the identical Cluster ion spectrometry (CIS) experiment, Ann. Geophys., 19, 1303-1354, 2001, http://www.ann-geophys.net/19/1303/2001/.

Sagdeev, R. Z. and Galeev, A. A.: Nonlinear Plasma Theory, New York: Benjamin, 1969.

Spangler, S. R.: Kinetic effects on Alfvén-wave nonlinearity, 1. Ponderomotive density fluctuations, Phys. Fluids, B 1(8), 17381746, 1989.

Spangler, S. R., Leckband, J. A., and Cairns, I. H.: Observations of the parametric decay instability of nonlinear magnetohydrodynamic waves, Phys. Plasmas, 4, 846-855, 1997.

Terasawa, T., Hoshino, M., Sakai, J.-I., and Hada, T.: Decay instability of finite-amplitude circularly polarized Alfvén waves: A numerical simulation of stimulated brillouin scattering, J. Geophys. Res., 91, 4171-4187, 1986.

Vasquez, B. J.: Simulation study of the role of ion kinetics in lowfrequency wave train evolution, J. Geophys. Res., 100, 17791792, 1995.

Vinaz, A. F. and Goldstein, M. L.: Parametric instabilities of circularly polarized large-amplitude dispersive Alfvén waves: excitation of obliquely-propagating electromagnetic daughter and side-band waves, J. Plasma Phys., 46, 107-127, 1991.

Wong, H. K. and Goldstein, M. L.: Parametric instabilities of circularly polarized Alfvén waves including dispersion, J. Geophys. Res., 91, 5617-5628, 1986. 